\title{
Novel Chipless RFID Technology Using Open Loop Resonator
}

\author{
Ayman S. Al-zayed ${ }^{*}$ and Shameena V.A \\ Electrical Engineering Department, Kuwait University, P.O. Box 5969, Al-Safat 13060, Kuwait \\ *Corresponding author
}

\begin{abstract}
The paper presents the design and development of a low cost compact chipless RFID system for secure near field data transfer. The proposed system is suitable for use in credit cards, personal data keeping cards and identification cards based on the principle of "data transfer by contact" which at present commonly uses the integrated circuits (IC) or magnetic strips. The proposed system consists of a compactable low cost RFID tag. The reader used in this technique is a simple waveguide.
\end{abstract}

\section{Keywords-chipless; OLR; RFID; uniplanar; waveguide}

\section{INTRODUCTION}

The radio frequency identification is presently became a widespread research area due to its tremendous applications. Commonly radio frequency identification (RFID) tags are used for item tracking. Another important uses of RFID is for personal identification. Generally these types of applications need a very high degree of security. Most conventional RFID application operates in the sense of proximate detections. Data transfer by contact can enhance decoding by contact enhances the security as the authorized person has to present himself before the detector with the tag. This contact can prevent the leak of critical data from the user's card. A good example using this principle exists in the detection using magnetic strips in automated teller machine (ATM) cards where the user swipes the card to decode the information. Thus magnetic strips together with their reader are widely employed by businesses and others to perform several identification functions. The main problems of these types of magnetic strip cards are the wear and tear. As the card or magnetic strip ages, the data stored in the card using the magnetic strip will be damaged which results in having to replace the non-functional card.

\section{DESIGN OF CHIPLESS RFID}

Various deigns of chipless RFIDs can be found in literature. The design of a chipless RFID system based on multiresonators is proposed in [1] which performs frequency signature encoding. The spectral signature is created by the transponders multi-resonating circuit which is a multi-stop band filter. The multi-resonator is a set of cascaded spiral resonators designed to resonate at particular frequencies and create stop bands. Since the design consists of antennas and transmission lines, this is not suitable in a near field set up. Also it requires a larger size for encoding larger number of bit. Another technique as mentioned in [2] is a completely passive and very low cost chipless RFID, consisting of arrays of capacitively-tuned microstrip dipoles, which absorb energy from a reader/interrogator. The tagged item ID is determined from the presence/absence of a set of predetermined resonance frequencies. Since data encoding is done by the presence and absence of capacitively tuned dipoles, this cannot be used to encode large number of data bit. The RFID system in [3] is based on using the wireless equivalent of optical barcodes. They consist of arrays of microstrip dipole-like structures that behave as resonant band pass or band stop filters tuned to predetermined frequencies. As multi bit read-only tags, the tagged item ID is determined from the presence/absence of a set of resonance frequencies. Hence, with $n$ barcodes in the field $(2 n-1)$ items can be identified. The number of bits of stored information is increased using multiple bands and accurately determining the center frequency of resonance for each barcode, and employing narrow bandwidth design resulting in arrays of low loss/high $\mathrm{Q}$ resonant strips. A biostatic detection system was set up based on network analyzer transmission coefficient $\left(\mathrm{S}_{21}\right)$ measurement is used to detect the barcodes. Two high gain patch antenna array were used as transmit and receive antennas and thus the system is not compact.

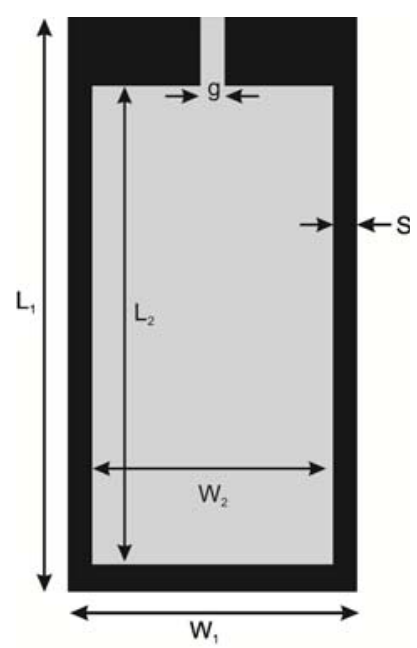

(a)

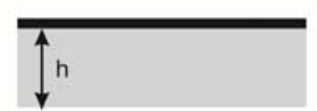

(b)

FIGURE I. GEOMETRY OF THE OLR BASED CHIPLESS RFID TAG (A) TOP VIEW AND (B) SIDE VIEW 
In this paper we present a tag which is simply an open loop resonator (OLR) printed on a substrate. This ORL will resonate at a particular frequency determined by the dimensional parameters of the structure. The main interesting thing in this design is the reliability of the structure. We can change the resonant frequency of the OLR by changing any of the dimensional parameters of the structure, and therefore, it is possible to generate many resonant frequencies or users by very minute change in the structure. The open loop resonator will absorb the electromagnetic energy from a transmission line (waveguide) with frequency corresponding to its resonance; consequently, this absorption will create a dip in transmission coefficient. This idea is used here to design chip less RFID tags. The structure of an OLR is shown in Figure 1. Geometrical parameters are given by $\mathrm{L}_{1}=30 \mathrm{~mm}, \mathrm{~L}_{2}=28 \mathrm{~mm}, \mathrm{~W}_{1}=10 \mathrm{~mm}$, $\mathrm{W}_{2}=9 \mathrm{~mm}, \mathrm{~g}=0.4 \mathrm{~mm}$, and $\mathrm{S}=1 \mathrm{~mm}$.

\section{THE WAVEGUIDE MEASURMENT SETUP}

As mentioned above, the data to be handled is in need of high degree of security and therefore the choice of a measurement technique is very critical. This paper presents a measurement setup which is simply a waveguide. The tag is inserted in the waveguide such that it is placed where the electric field is maximum (at the centre of broad dimension of wave guide). This is shown in Figure 2. The insertion of the OLR printed tag will create a dip in the $S_{21}$ which is due to the absorption of that particular frequency by the OLR. Since the tag is inserted inside the waveguide there will be no leakage of data and thus a system with high security is achieved.

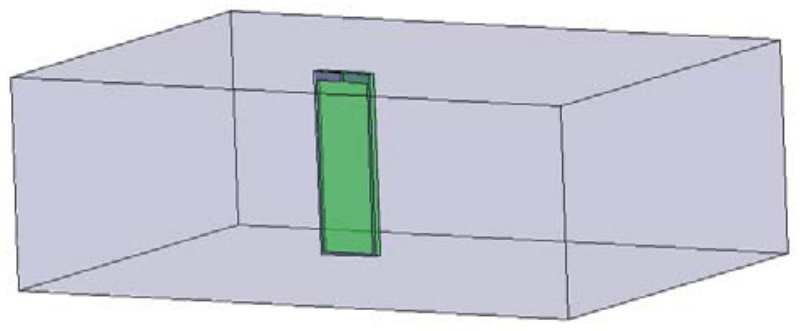

FIGURE II. SIMULATED WAVEGUIDE MEASURMENT SET-UP

The transmission coefficients of matched waveguide, matched waveguide loaded with substrate and matched waveguide loaded with open loop resonator printed substrate above the cutoff frequency of the wave guide are shown in Figure 3.

From the figure it is clear that a waveguide loaded with open loop resonator printed substrate will produce a high level of attenuation in the transmission coefficient. This dip is produced due to the absoprption of electromagnetic energy by the open loop resonator. Since the frequency response has high quality factor we are able to generate a large number of different tags whose null frequencies are seperated by a narrow frequency guard band by varying any one of the dimensional parameter of OLR, and therefore many users can be accomidated.

Variation of transmission coefficient with one of the parameter $L_{2}$ of OLR is shown in Figure 4. From the figure it can be noticed that as the parameter $\mathrm{L}_{2}$ increases, the resonant (Dip) frequency decreases. It is also obvious from the figure that variation in resonant frequency is very narrow with variation in $L_{2}$. This is due to the high quality factor of the propused design.

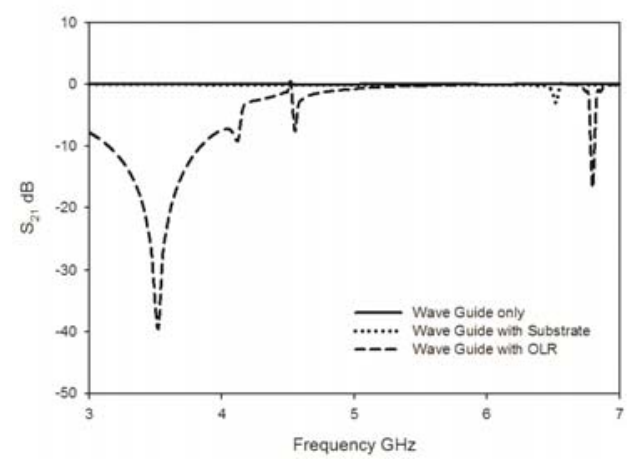

FIGURE III. SIMULATED TRANSMISSION COEFFICIENTS OF MATCHED WAVEGUIDE, MATCHED WAVEGUIDE LOADED WITH SUBSTRATE AND MATCHED WAVEGUIDE LOADED WITH OLR PRINTED SUBSTRATE

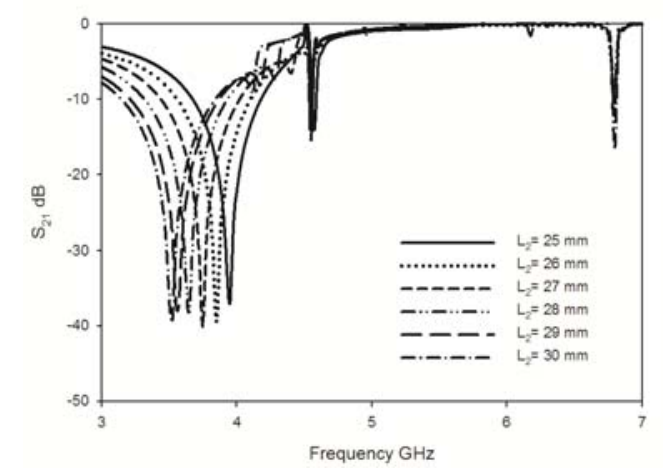

FIGURE IV. VARIATION OF TRANSMISSION COEFFICIENTS WITH LENGTH $\mathrm{L}_{2}$ OF OLR

The variation of reflection coefficient frequency with a very small change in $\mathrm{L}_{2}$ parameter of the open loop resonator is shown in Figure 5. It is understood from the figure that fine variation in length will create a shift in the resonant frequency of OLR and thus it is very easy to produce different absorption frequncies with a guard band even less than $1 \mathrm{MHz}$. Using this technique we can produce more than 4500 different frequencies (different tags) in the feruency range of $2.5 \mathrm{GHz}$ to $7 \mathrm{GHz}$. So it is very easy to code 12 bits of data using this technique.

\section{EXPERIMENTAL METHODOLOGY}

Figure 6 show the photograph of the fabricated OLR and Figure 7 shows the experimental setup using a network analyzer and a waveguide.

A slotted waveguide of dimension $7 \mathrm{~cm} \times 3.5 \mathrm{~cm}$ is used. A slot is there on the top surface of the waveguide. The tag is inserted at the center where the electric field is maximum for 
the $\mathrm{TE}_{10}$ mode, and therefore, maximum interaction between tag and electric field will take place.

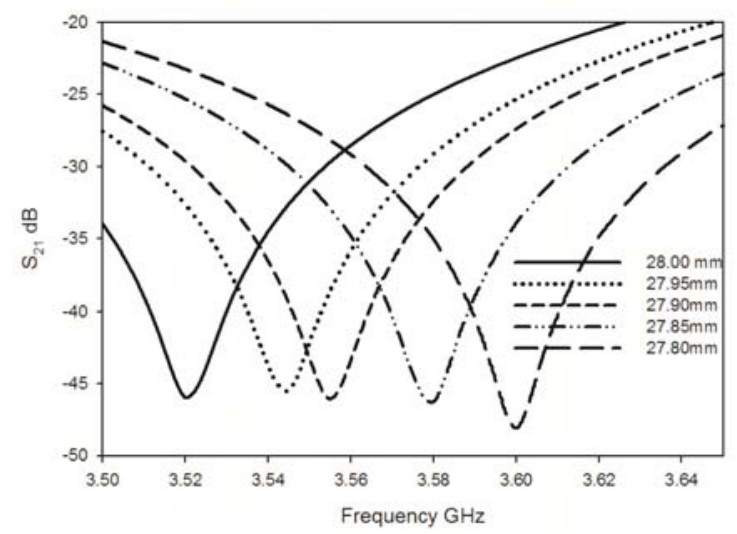

FIGURE V. VARIATION OF REFLECTION COEFFICIENTS WITH VERY SMALL CHANGE $\mathrm{L}_{2}$

Measured and simulated transmission coefficient of a tag with OLR loaded waveguide with dimensional parameters as shown in Figure1 is shown in Figure 8. From the figure it is clear that the simulated and experimental results are in good agreement.

The next step involved is the preparation of the look-up table containing the changes in resonant frequency due to the insertion of the tag and the corresponding binary code. If a guard band of $1 \mathrm{MHz}$ is chosen. The code of tag in decimal number can be obtained as:

$$
\text { Code }=\left(\text { Resonant Frequency in } \mathrm{GHz}-\mathrm{f}_{1}\right) / 0.001
$$

where first frequency $\mathrm{f}_{1}=2.9 \mathrm{GHz}$.

The above equation for calculating the code is valid when the tag is constructed on an FR4 substrate of length $22 \mathrm{~mm}$, width $30 \mathrm{~mm}$, thickness $1.6 \mathrm{~mm}$, and dielectric constant 4.4 . The first frequency $\mathrm{f}_{1}$ is the frequency corresponding to the tag with an OLR of resonant frequency $2.9 \mathrm{GHz}$.

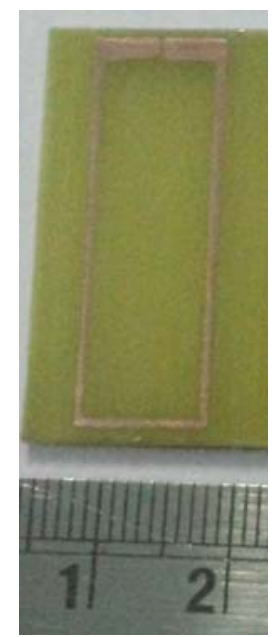

FIGURE VI. PHOTOGRAPH OF THE FABRICATED OLR

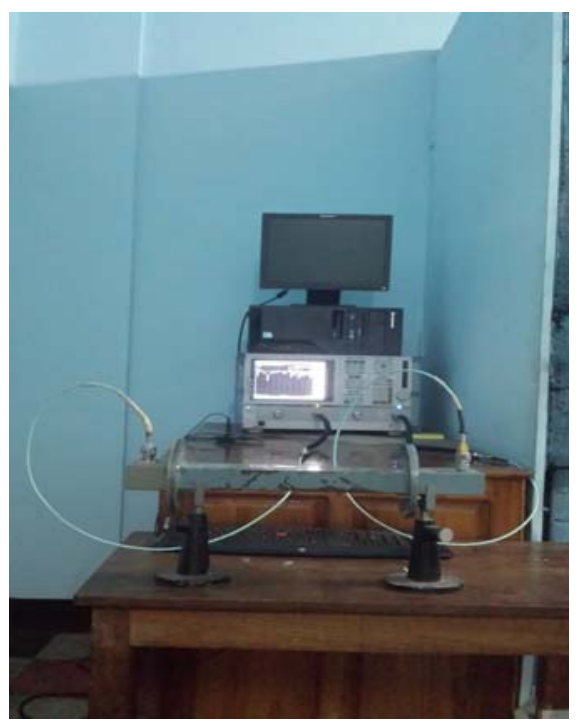

FIGURE VII. EXPERIMENTAL SETUP FOR WAVEGUIDE MEASUREMENT

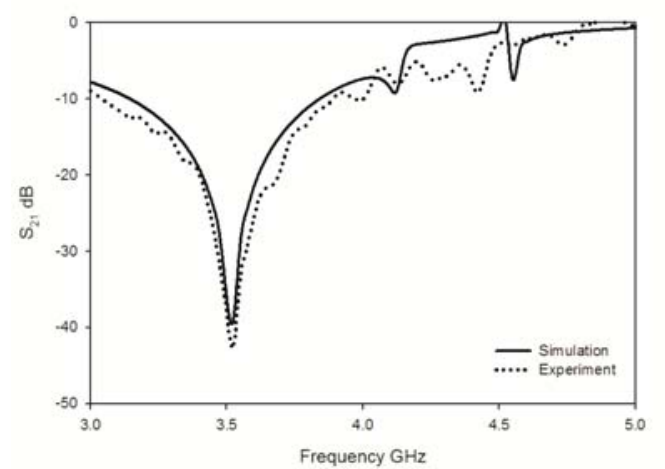

FIGURE VIII. SIMULATED AND MEASURED TRANSMISSION COEFFICIENTS OF WAVEGUIDE LOADED WITH TAG WITH DIMENSIONS SHOWN IN FIGURE 1

\section{CONCLUSION}

The paper presents the design and development of a new technique for the use in chipless RFIDs for bank cards and similar devices. It is easily possible to code 12 bits within the conventional credit card dimensions even by using the amplitude of the signals. The methodology is found be well suited for low cost RFID applications with moderate data requirements.

\section{REFERENCES}

[1] S. Preradovic, I. Balbin, N. C. Karmakar, G. Swiegers, “A Novel chipless RFID system based on planar multiresonators for barcode replacement,” Proc. 2008 IEEE RFID Conference, April 16-17, 2008, Las Vegas, Nevada, USA, pp. 289-296.

[2] I. Jalaly, I.D. Robertson, “Capacitively-tuned split microstrip resonators for RFID barcodes,” European Microwave Conference 2005, Vol. 2, 4-6 Oct. 2005.

[3] I. D. Robertson, "RF barcodes using multiple frequency bands," IEEE MTT-S Digest, June 2005. 